\title{
Optimal generalization of digital elevation model constrained by granularity measure
}

\author{
Timofey Samsonov ${ }^{\mathrm{a}, *}$ \\ ${ }^{a}$ Lomonosov Moscow State University, Faculty of Geography, Department of Cartography and Geoinformatics, Moscow, Russia, \\ tsamsonov@geogr.msu.ru, \\ * Corresponding author
}

Keywords: cartographic relief presentation; digital elevation model; DEM; generalization

\begin{abstract}
:
Digital elevation models (DEMs) are widely used for cartographic relief presentation, most commonly in a form of hypsometric tints or hill shading. Increasingly precise and detailed public DEMs are released almost every year, thanks to the current progress in remote sensing and other terrain data acquisition techniques. Recent advancements in the coverage, quality and resolution of global DEMs facilitate the overall improvement of detail and reliability of terrainrelated research. Improvement of quality is commonly believed to go hand-in-hand with increasing DEM resolution and detail. However, in many cases the opposite is true, and appropriate DEM generalization is needed. Geographic problem solving is conducted in a wide variety of scales, and the data used for mapping must have the corresponding level of detail. Unfortunately, with widespread accessibility of detailed DEMs this principle is often violated, and the data is used for mapping at scales far smaller than what is appropriate.
\end{abstract}

Existing coarse-resolution global DEMs are not suitable for generating high-quality cartographic relief representations, since they are undergeneralized and accumulate maximum possible information in every pixel. It is clear that guidelines and effective principles for DEM generalization at small scales are needed. Specifically, it is unclear when to stop generalization of DEM surface if some automated procedure is applied to solve this task. Simplistic error-based measures like vertical distance are not effective for cartography, since they do not account for morphology of the surface perceived by map reader.

The current research is dedicated to solution of this problem. First, a new DEM granularity measure which reflects the typical width of a landform represented in DEM surface is introduced. Second, the optimal granularity is statistically learned using DEMs reconstructed from multiple fragments of 1:200 000, 1:500 000 and 1:1 000 0000 topographic maps covering different terrain conditions. Finally, it is demonstrated how granularity measure can be used as a constraint during DEM generalization to achieve the desired level of detail.

The work has been funded by Russian Science Foundation (RSF) grant No 19-77-00071. 